\title{
Vaginal Robotic Supracervical Hysterectomy in an Ovine Animal Model: The Proof of Concept
}

\author{
Jonia Alshiek ${ }^{1,2}$, Liron Bar-El ${ }^{3 *}$, S. Abbas Shobeiri, ${ }^{1,4}$ \\ ${ }^{1}$ Department of Obstetrics \& Gynecology, INOVA Women's Hospital, Falls Church, VA, USA \\ ${ }^{2}$ Department of Obstetrics \& Gynecology, Hillel Yaffe Medical Center, Hadera, Israel \\ ${ }^{3}$ Department of Obstetrics and Gynecology, Lenox Hill Hospital/Northwell Health, New York, USA \\ ${ }^{4}$ Department of Bioengineering, George Mason University, Fairfax, VA, USA \\ Email: \#Abbas.Shobeiri@inova.org
}

How to cite this paper: Alshiek, J., Bar-El, L. and Abbas Shobeiri, S. (2019) Vaginal Robotic Supracervical Hysterectomy in an Ovine Animal Model: The Proof of Concept. Open Journal of Obstetrics and $G y$ necology, 9, 1114-1129.

https://doi.org/10.4236/ojog.2019.98108

Received: June 9, 2019

Accepted: August 6, 2019

Published: August 9, 2019

Copyright $\odot 2019$ by author(s) and Scientific Research Publishing Inc. This work is licensed under the Creative Commons Attribution International License (CC BY 4.0).

http://creativecommons.org/licenses/by/4.0/ Open Access

\begin{abstract}
Objective: To gain insight into the feasibility and safety of a novel vaginal robot for performing supracervical hysterectomy in an ovine model. Introduction: The clinical application of transvaginal natural or fice transluminal endoscopic surgery (vNOTES) has broadened significantly. vNOTES reduces wound complications such as infection, hematoma formation, or herniation and is currently utilized for hysterectomy, adnexal surgery, myomectomy, and staging surgery for endometrial cancer. Robotic assistance has been proposed to overcome the current vNOTES mechanical obstacles. The implementation of the current robots has limited utility due to their bulk and inflexibility. Robotic Natural Orifice Transluminal Surgery (rNOTES) is the new frontier in advancement of surgical robots. In developing new task specific robots, it is important to utilize an accurate model for testing. A novel vaginal robot introduced through the posterior cul-de-sac to perform a complete retrograde hysterectomy is the subject of this study. Methods: The study was conducted at the animal lab, Asaf-Harofe hospital, Israel. The ovine model was preferred since the anatomical landmarks and vascular anatomy are comparable to the human except for a bicornuate uterus in sheep. A vaginal robotic supra-cervical hysterectomy was performed in a sheep. Results: A vaginal robotic supracervical hysterectomy was performed successfully in an ovine model. The uterus was extracted via the entry point in the pouch of Douglas. $20 \mathrm{cc}$ of blood loss was reported and no complications were observed. Conclusions: Vaginal supracervical hysterectomy via a vaginal approach using a novel robotic system was found to be feasible.
\end{abstract}

\section{Keywords}

Hysterectomy, Robot, Supracervical, Robotic, Vaginal, vNOTES

${ }^{*}$ Dr. Liron-Bar-El has made equal primary author contribution to development and production of this manuscript. 


\section{Introduction}

\subsection{Vaginal Approach}

Recent technologic advances in endoscopic instrumentation and optics have allowed the development of a less invasive alternative to conventional laparoscopic surgery. The introduction of minimally invasive surgery has promoted the development of new surgical techniques, including the natural orifice transluminal endoscopic surgery (NOTES). NOTES yields access to the abdominal cavity without any incisions on the abdominal wall (scarless surgery), and the natural orifices of the body surface, such as the mouth and the vagina, served as the gateway to the peritoneal cavity [1] [2]. There are some advantages of this technique over open and conventional laparoscopic surgery, including avoidance of incision-related complications (wound infections, adhesions, and hernias), less postoperative pain, improved aesthetics, and a diminished immunologic impact [3].

Recently, clinical application of transvaginal NOTES has broadened significantly in gynecology. Using transvaginal NOTES, not only adnexal surgery and hysterectomy, but also myomectomy and oncologic surgery could be performed safely and effectively in selected patients [4]. The first case series of pure transvaginal NOTES for adnexal diseases was reported by Lee et al. [5] in 2012. Other groups [6] [7] [8] have also described their initial experience with transvaginal NOTES using a single port and concluded that transvaginal NOTES was a feasible and effective surgical technique in properly selected female patients with adnexal masses. Hysterectomy via the vaginal route was first reported by Su et al. [9] in the same year; however it was Baekelandt et al. [10] that reported the first total vNOTES hysterectomy (TVNH), in which the entire procedure was performed using transvaginal NOTES under the pneumovagina.

Clinical contraindications are active infections, pregnancy or cervical cancer. Technical difficulties may be encountered in the case of previous gynecologic surgery, gynecologic infections, or previous inflammatory digestive diseases (such as inflammatory bowel disease, diverticulitis or appendiceal peritonitis) as they may lead to the formation of dense adhesions in this region. The presence of endometriosis should also be ruled out as these lesions are usually located posterior to the uterus, either in uterosacral ligaments, in the retrocervical region or in the rectovaginal septum. This prevents access to the pelvic cavity through the posterior fornix of the vagina due to the high risk of iatrogenic injuries of adjacent organs during the creation of the access to the pelvic cavity. Another point to consider is the practice of transvaginal surgery in a young nulliparous patient as the impact of transvaginal surgery on fertility is yet unknown [11].

\subsection{Robotic Assistance}

The biggest drawback of the vNOTES laparoscopy is the inability to explore the entire peritoneal cavity, especially the anterior uterine wall and the peritoneum 
covering the surface of the bladder and broad ligaments [12]. The NOTES approach for gynecologist surgeries could be further and more comfortably applied if the tools used had better maneuverability and increased degree of freedom as provided by flexible robotic arms.

The first robot-assisted NOTES hysterectomy was published by Lee et al. [13] in 2015, using the vaginal working channel established by inserting a single-site multi-instrument silicon port. The operation began as in conventional vaginal surgery, with resection of the vaginal wall around the cervix. Anterior and posterior colpotomy were performed and the uterosacral ligaments and uterine vessels were dissected. The patient-side cart of the da Vinci Surgical System was then placed between the patient's legs, and each responsible port was docked onto the assigned robotic arms. Robot-assisted NOTES hysterectomy was performed after achieving adequate pneumoperitoneum and the uterus was morcellated through the vagina. They found that the robot-assisted NOTES technology allowed the surgeon to reach deeper places, but that its implementation was limited by the lack of appropriate instrumentation, which required further development and breakthrough. Therefore, they concluded that at this stage robot assisted vNOTES was useful only for limited applications in highly selected patients.

\subsection{Animal Model for Vaginal Surgeries}

Vaginal surgery research and training has been historically preformed on cadavers. This has proved ineffective, because non-living fresh frozen specimens either lack laxity or may be too lax. Low-fidelity vaginal hysterectomy training simulators have also developed and provided a useful introduction to vaginal hysterectomy; however, they have proved inadequate and unrealistic. Hence, an anatomically similar, live animal model is currently the preferred method for vaginal surgical studies and training [14].

The ovine model is currently the preferred animal model for vaginal and pelvic surgeries since the anatomical landmarks and vascular anatomy are most comparable to the human female (Figure 1) [15]. A comprehensive study that aimed to compare the anatomy of the sheep with female pelvis argued that although sheep are quadrupeds, the gross and microscopic anatomies are similar to the female pelvis [16]. Principal differences are the shape of the uterus (bicornuate) and its orientation, the absence of the sacrospinous ligament and the internal obturator. The levator ani (except for the puborectalis) and a more developed coccygeus muscle are present - coinciding with the tail. The dimensions and morphology of the ovine vagina is comparable to humans and the retropubic and the rectovaginal space are accessible transvaginally (Figure 2). The study concluded that the ovine pelvic floor has many anatomical and ultrastructural similarities to the female pelvic floor and is appropriate as a model for gynecologic surgeries [16]. The vascular anatomy of the female human and ovine pelvis have striking similarities (Figure 3 ). 

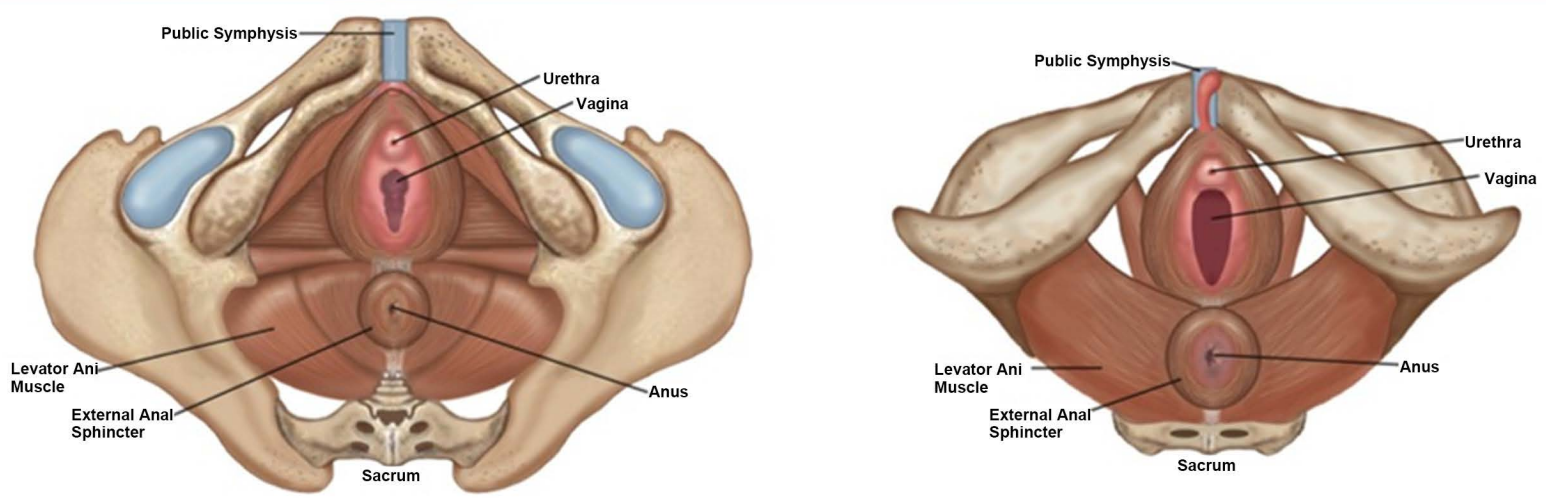

Figure 1. Axial comparative view of ovine vs female human pelvis.
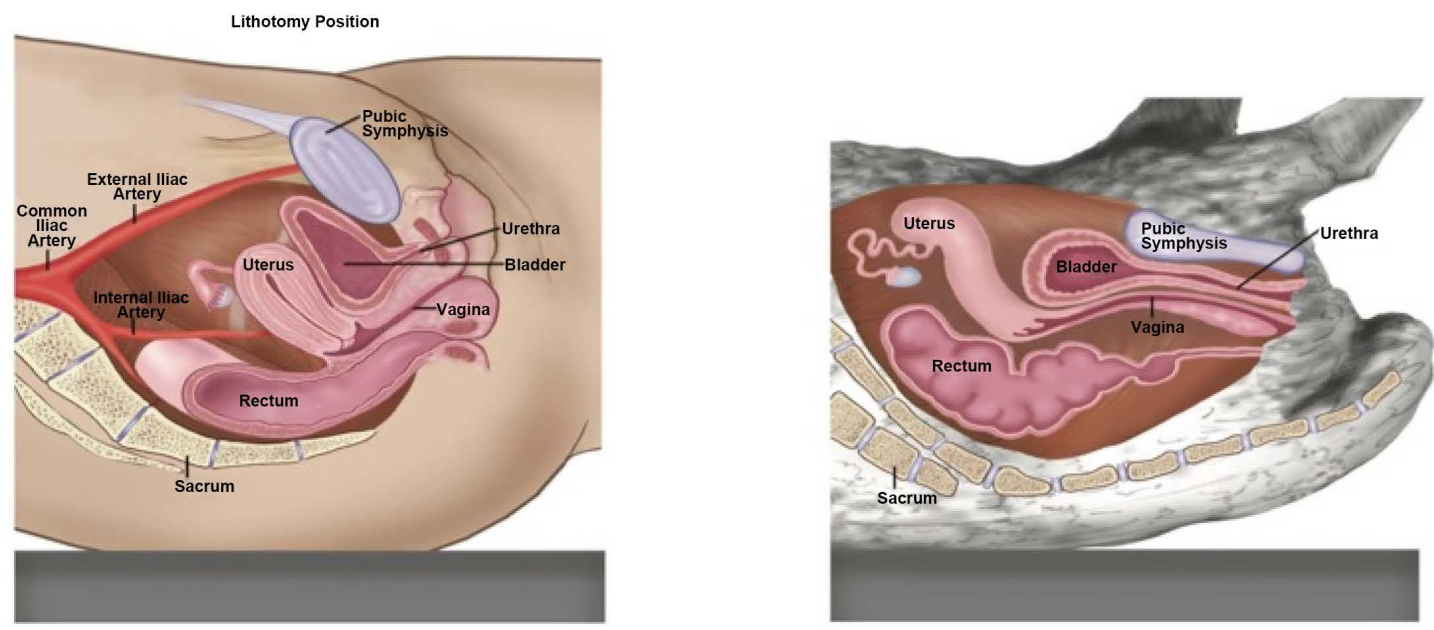

Figure 2. Sagittal comparative view of ovine vs female human pelvis.

Many studies have used the ovine model for vaginal surgeries because the ovine model best resemble the female human pelvis. In a study done in 2017, twenty-nine postgraduate surgeons performed transvaginal meshes, sacrospinous ligament suspension, and OASI repair in sheep as compared with human cadavers. The study showed a significant superiority of sheep over human cadavers for all items evaluated regarding surgical dissections. Two vaginal hysterectomies were also performed in which operating time, surgery, and anatomy were nearly identical to that of humans [15]. Another study demonstrated that training in vaginal surgery for pelvic organ prolapse (POP) is feasible and useful in an animal model and concluded that the sheep is the preferable model [17]. Similarly, a study describing transvaginal mesh insertion in the ovine model stated that the anatomical spaces and elements were easily identified- allowing for the mimicking of surgical procedures performed in women suffering from pelvic organ prolapsed [18]. Multiple studies on sheep model for uterine 

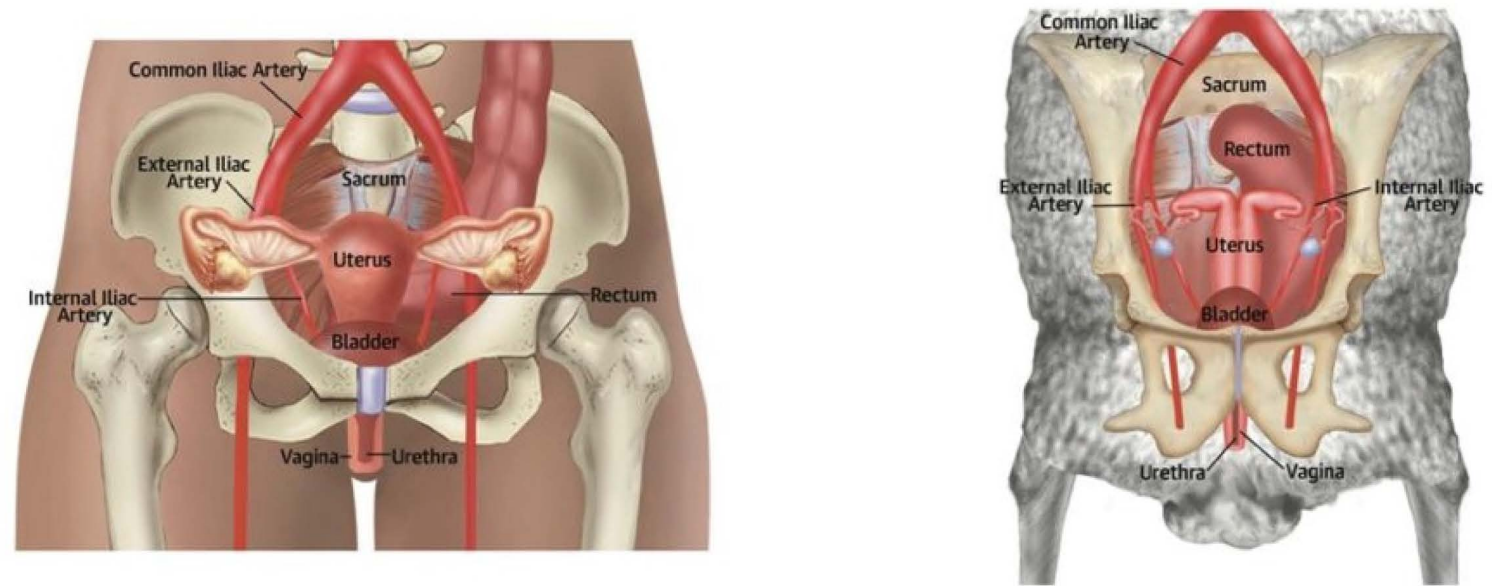

Figure 3. Coronal comparative view of ovine vs female human pelvis.

transplantation were performed, all mentioning the unequivocal choice of an ovine model [19] [20] [21] [22] [23]. An ovine model was also used to demonstrate the feasibility of intrauterine repair of myelomeningocele using the da Vinci ${ }^{\oplus}$ System [24].

\subsection{Total vs. Supracervical Hysterectomy}

Supracervical hysterectomy has become a popular option in presence of normal cervical cytology. Hysterectomy involves removal of the uterine corpus and cervix (total hysterectomy) or removal of the uterus with preservation of the cervix (supracervical or subtotal hysterectomy). The only absolute indications for cervical removal at the time of hysterectomy are suspected gynecologic malignancy and its precursors. Relative indications may include uterine prolapse and cervical or retrocervical endometriosis [25] although increasingly supracervical hysterectomy is performed at the time of sacrocolpopexy for pelvic organ prolapse due to decreased risk of mesh extrusion.

Proponents of supracervical hysterectomy suggest multiple advantages but a comprehensive literature review finds no significant differences in sexual function or no high-quality studies to substantiate the benefits of supracervical vs. total hysterectomy for benign gynecologic indications. Randomized controlled trials that compared complications of supracervical abdominal hysterectomy (SAH) to other modalities found no significant difference for most postoperative complications (urinary tract injury, pain score, wound infection, persistent pain after discharge, bowel obstruction, or pelvic organ prolapse) [25] [26] [27] [28] [29]. The early postoperative complications were also similar [30] [31]. It has been suggested that supracervical hysterectomy carries a decreased risk of pelvic organ prolapse but there are no randomized or long-term data to support or refute that argument [26]. Improved sexual function after surgery was also sug- 
gested as an advantage of laparoscopic assisted supracervical hysterectomy (LASH) over total laparoscopic hysterectomy (TLH) [32]. This difference was hypothesized to be associated with vaginal scar tenderness, shortening of the vagina, absence of the cervix, disruption of vaginal and cervical innervation, and vaginal dryness [33] [34]. A randomized controlled trial showed no significant difference in sexual or psychologic functioning postoperatively when the two procedures were compared [35]. Compared with total abdominal hysterectomy, supracervical abdominal hysterectomy ( $\mathrm{SAH}$ ) was thought to be associated with lower rates of urinary dysfunction because of maintenance of normal bladder innervation and preservation of the upper vagina support structures; but a meta-analysis of TAH and SAH did not support this theory [36].

Notwithstanding the above, a Cochrane review found reduced febrile morbidity in patients undergoing supracervical hysterectomy when compared with total hysterectomy [37]. The same review showed a non-significant decreased rate of intra-abdominal hematoma or abscess after supracervical hysterectomy. A possible explanation for these findings is decreased dissemination of vaginal flora through the peritoneum and abdominal wound when the cervix is left in situ. While this may also be the case for LASH, similar randomized clinical trials have not been performed in the laparoscopic setting. Reduced operating time, blood loss and reduced recovery time were also suggested as advantages of LASH over TLH.

Opponents of LASH mention the future risk of cervical malignancy as a downfall, however with the availability of excellent cervical screening programs, removal of the cervix to prevent malignancy has been reported as unjustified [38]. The risk of development of cervical stump neoplasia in women with previously normal smear test results is $0.3 \%$ [39] and is further reduced to, $0.1 \%$ with ongoing screening [25]. The possibility of ongoing cyclical bleeding was also presented as a drawback. The incidence of cyclical vaginal bleeding after LASH varies widely, from $0 \%$ to $25 \%$ [26]. This range likely reflects the completeness of follow-up, the definition used for bleeding, and the level of amputation of the uterine corpus. Ideally, amputation of the cervix is at the level of the internal os, so that the isthmic portion of the uterus is removed along with the endometrium, thereby minimizing the likelihood of future cyclical bleeding. In addition to postoperative cyclical bleeding, a separate concern about residual endometrium after LASH is the risk of subsequent endometrial cancer, that is increased if the woman receives estrogen therapy without progesterone [40].

The removal of the uterus after its resection is another consideration. in TLH, the uterus may be removed intact vaginally or via morcellation. At LASH, intact removal requires a colpotomy, and therefore morcellation is the primary method of specimen retrieval. Evidence from case reports suggests that iatrogenic endometriosis may complicate hysterectomy with associated uterine morcellation [26]. Furthermore, Incomplete retrieval of fragments of myometrium and leiomyomas after morcellation may lead to peritoneal leiomyoma seedlings [41] [42] [43] [44]. 
Supracervical hysterectomy has been performed for almost as long as total hysterectomy via both laparotomy and laparoscopy. Although there are strong proponents both for and against LASH, there are no RCTs that demonstrate clinical superiority of any surgical technique for any outcomes. Performance of supracervical vs. total laparoscopic hysterectomy is therefore dependent on the desire of both the patient and the surgeon in an appropriate clinical setting.

In summary, conventional vaginal surgery has been used in gynecologic practice to avoid the disruption of the abdominal wall. Recently, the clinical application of vNOTES has broadened significantly. This technique appears to reduce wound complications after surgery, such as infection, hematoma formation, or herniation. The vNOTES approach is currently adopted for hysterectomy, adnexal surgery, myomectomy, and staging surgery for endometrial cancer [45] [46] [47]. The feasibility and safety of supracervical hysterectomy through vNOTES, was recently confirmed [48]. New technology is needed to overcome the current mechanical obstacles in preforming gynecologic laparoscopic surgeries via vNOTES. Robotic assistance has been proposed for that matter. The implementation of the available robots is limited by the lack of appropriate instrumentation, the massive robot's corpus, a time-consuming docking set-up and the need for initial vaginal colpotimies prior to the robotic surgery.

In this study we aim to perform the first robotic vNOTES supracervical hysterectomy on an ovine model using the novel Hominis ${ }^{\mathrm{Tm}}$ surgical system(Memic Innovative Surgery, Or Yehuda, Israel) (Figure 4). Since no superiority was proven for total hysterectomy for any outcome, we chose to perform a supracervical hysterectomy and remove the intact uterus transvaginally through the robot trocar without the need for morcellation. The procedure aims to demonstrate the Hominis ${ }^{\text {Tx }}$ surgical system's efficiency and safety in preforming pelvic surgeries in a scarless fashion and with no need for prior surgical procedures.

\section{Methods}

The study was conducted at the animal lab, Asaf-Harofe hospital, Israel and was approved by the Ethics Committee of Asaf-Harofe hospital (Authorization number 15/2018).

A healthy mature ewe was used as a model. Food was withdrawn 24 hours before the procedure. Institutional guidelines for the care of experimental animals were followed. The senior author (SAS) who had significant prior robotic experience, completed successful completion of dry simulation exercises (Figure 4) prior to undertaking the Ovine vaginal robotic supracervical hysterectomy.Dr. S. A. Shobeiri and Dr. J. Alshiek also trialed the vaginal trocar for the robotic system in the dry lab at INOVA Advanced Surgical Technology \& Education Center (Falls Church, VA). The procedure was performed on one ovine model via a remote console through video guidance (Figure 5). Since this was a feasibility study, no controls were involved. Estimated blood loss and any difficulties with the procedure were tabulated. 


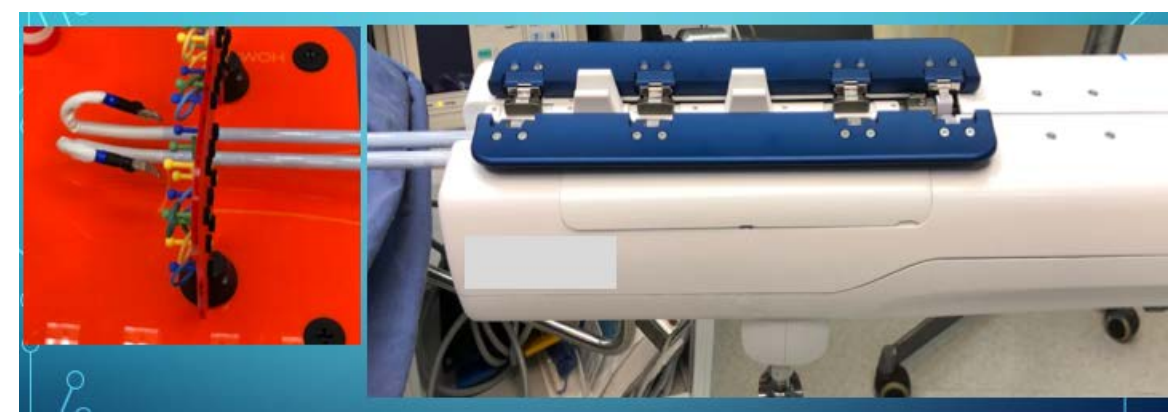

Figure 4. The vaginal robotic system during a dry simulation exercise. The arms are truncated in this picture to fit the space.

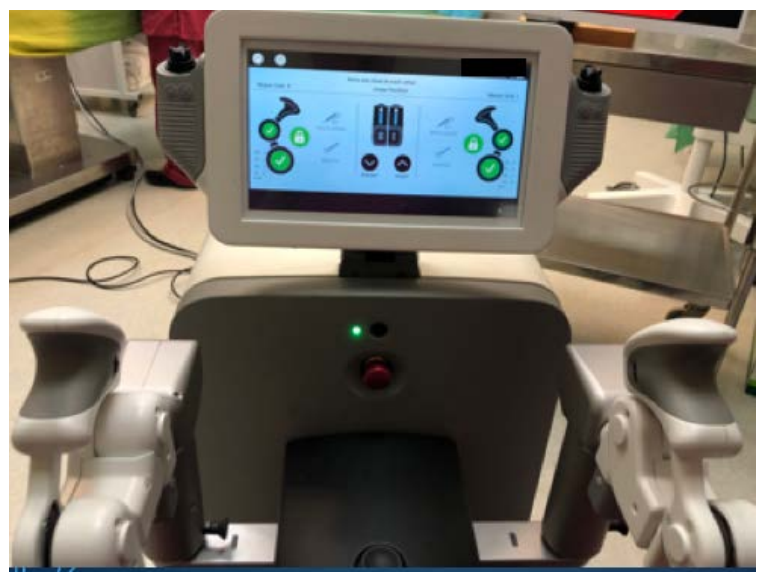

Figure 5. The vaginal robot operating console during dry exercises.

\section{Results}

A vaginal robotic supracervical hysterectomy was performed successfully in an ovine model as detailed below:

The ewe underwent general anesthesia using intramuscular midazolam $(0.5$ $\mathrm{mg} / \mathrm{kg}$, pre-anesthetic), propofol (5 mg/kg) and isoflurane (1.5\%) in 100\% oxygen with a continuous infusion of fentanyl $(10 \mathrm{mg} / \mathrm{kg} / \mathrm{min}, \mathrm{iv})$ and a neuromuscular blockade with pancuronium $(0.05 \mathrm{mg} / \mathrm{kg}$, iv). The ewe was subjected to mechanical ventilation with a $10 \mathrm{~mL} / \mathrm{kg}$ adjustment of the respiratory rate to maintain the exhaled concentration of $\mathrm{CO}_{2}$ between 35 and $45 \mathrm{mmHg}$.

After general anesthesia was applied, the ewe was laid in lithotomy position resembling that assumed by women on the operating table and the urinary bladder was catheterized through the urethra with a Foley catheter. A $5 \mathrm{~mm}$ laparoscopic port was inserted under vision in the mid abdomen. The abdomen was then inflated to $15 \mathrm{mmHg}$ and another $5 \mathrm{~mm}$ lateral laparoscopic port was inserted.

After entry and successful insufflation, the pelvis and abdomen were inspected, with a special focus on the anatomy of the ureter, the presence or absence of disease, and the visualization of the cul-de-sac. The ewe was placed in 30-degree lithotomy position to afford visualization of the cul-de-sac. 
A designated vaginal trocar of the robot system was used for a direct entry into the Pouch of Douglas (Figure 6). The trocar uses an introducer guide followed by a blunt conduit tube through a vaginal GelPOINT port (Applied Medical, Ranco Santa Margarita, CA, USA). A successful entry into the Pouch of Douglas was performed without a need for prior colpotomy (Figure 7). The trocar insert was removed leaving the channel open (Figure 8) for introduction of the robotic arms into the vagina (Figure 9). The robot was secured on designated arms facing the ewe's vagina and the Hominis ${ }^{\text {Th }}$ surgical arms were inserted through the vaginal port.

The robotic arms were retroflexed in position to perform a supracervical hysterectomy following the conventional steps of laparoscopic hysterectomy: The infundibulopelvic ligament was coagulated and cut (Figure 10) with the use of the energy source of the robotic arms; the round ligament and the remainder of the broad and cardinal ligaments were divided (Figure 11); the vesicouterine peritoneum was dissected off the anterior portion of the uterus, and the uterine arteries were skeletonized. At this point, the ascending branch of the uterine artery was identified, cauterized, and divided. The cervix was then amputated from the corpus at a point just below the internal cervical os and superior to the uterosacral ligaments (Figure 12). The robotic arms were straightened, and the uterine corpus was extracted via the vaginal entry point in the pouch of Douglas, with no need for morcellation (Figure 13). The vaginal cup was closed with continuous sutures. On average $20 \mathrm{cc}$ of blood loss was reported, no complications were observed, and no technical difficulties were encountered during surgery.

\section{Discussion}

The aim of the current study was to perform a robotic vaginal supracervical hysterectomy on an ovine model using the novel Hominis ${ }^{\text {Tit }}$ robotic system. Since supracervical hysterectomy is becoming more common in women to preserve pelvic floor support, we chose to perform a supracervical hysterectomy and remove the intact uterus transvaginally without the need for morcellation.

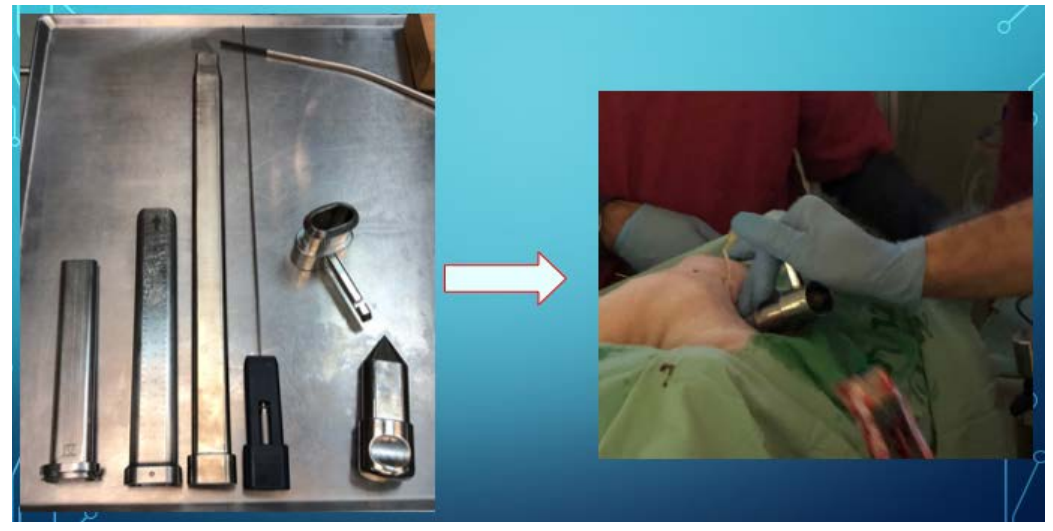

Figure 6. The trocar assembly for vaginal insertion (left); The trocar inserted in the Ovine vagina (right). 


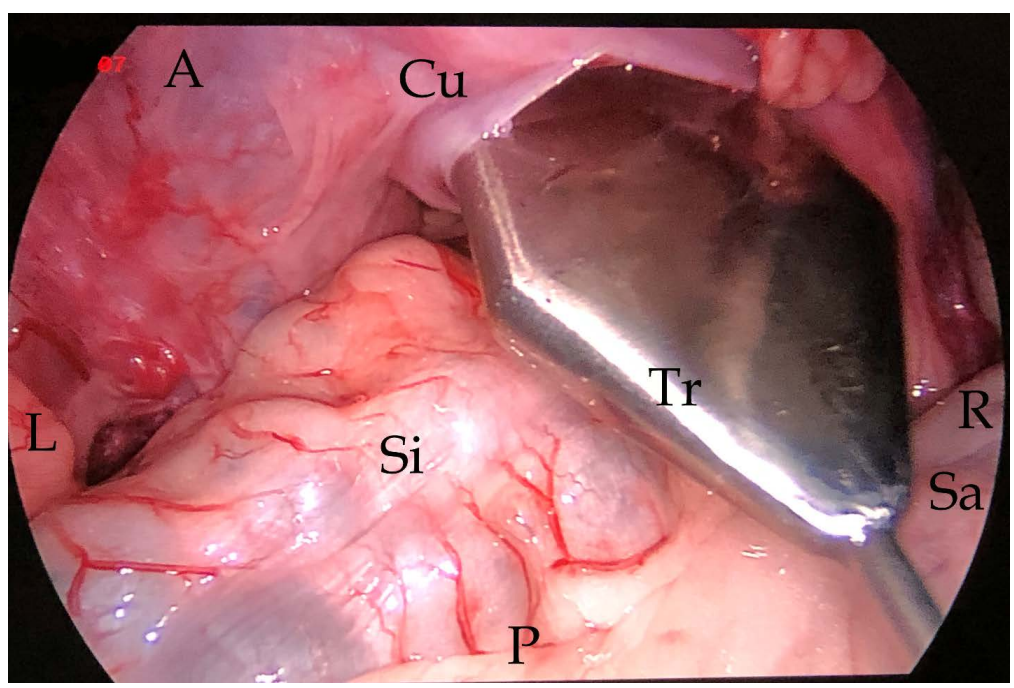

Figure 7. Trocar transvaginal entry into the cul-de-sac.

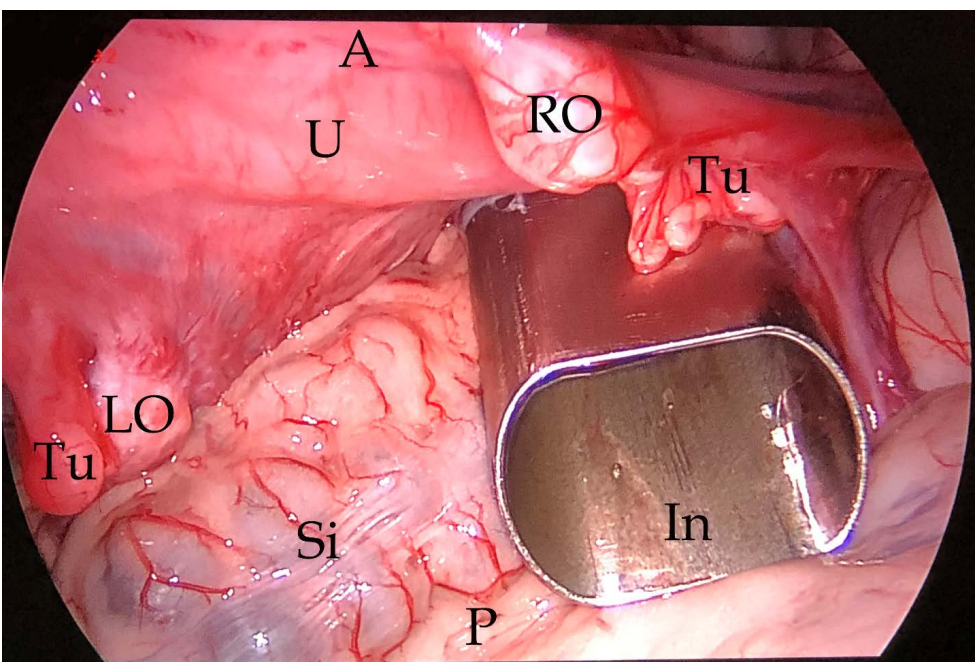

Figure 8. Trocar insert removed in preparation of the robot entry.

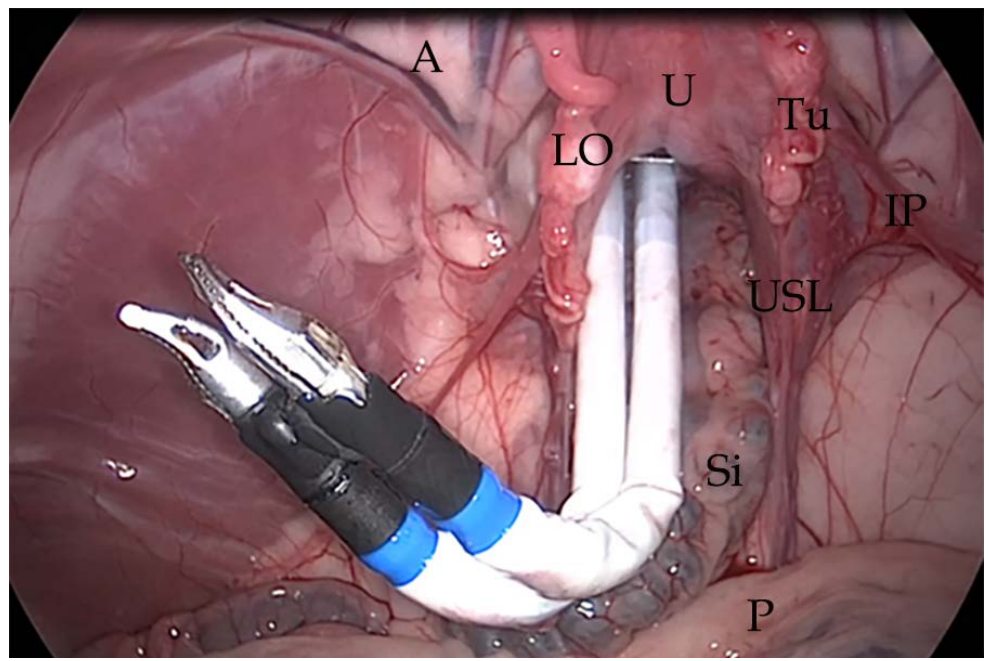

Figure 9. Robot arms introduced into the peritoneal cavity. 


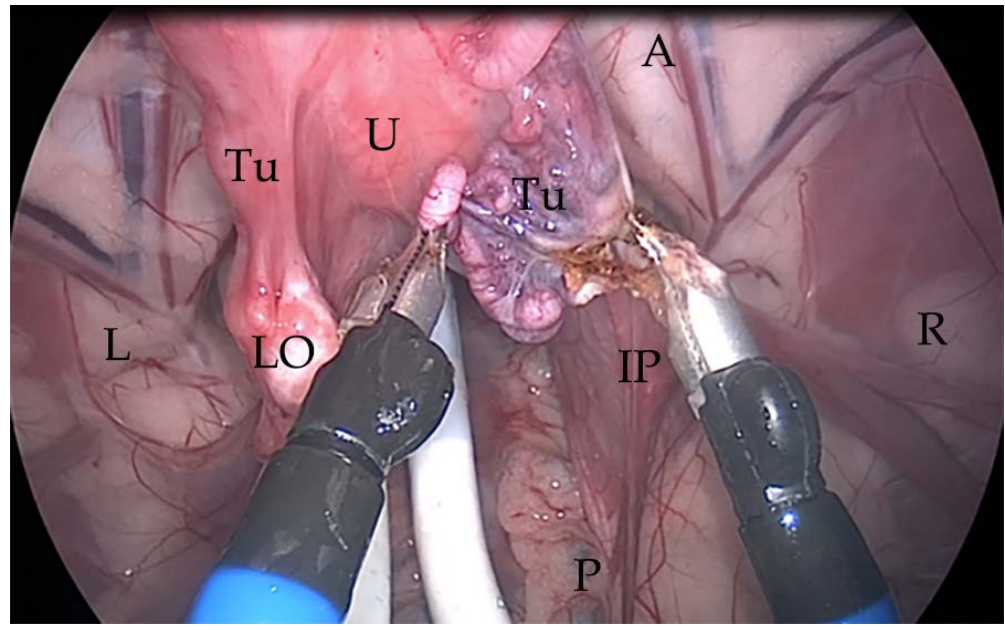

Figure 10. Initiation of the robotic hysterectomy by folding the robotic arms and coagulating and cutting the infundibulopelvic ligament on the right.

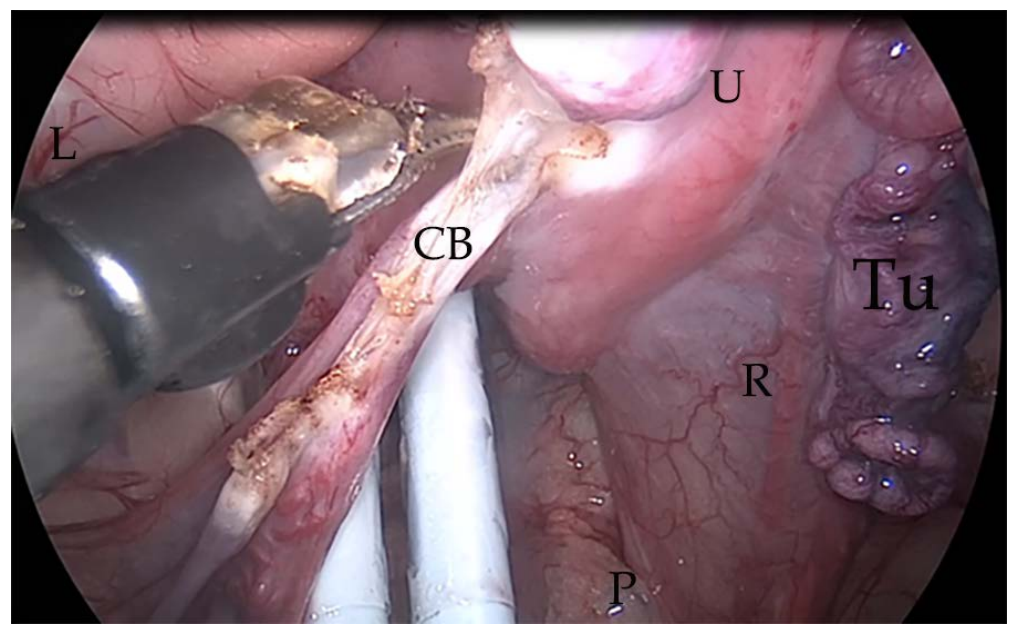

Figure 11. The coagulation and cautery of the right cardinal and broad ligaments.

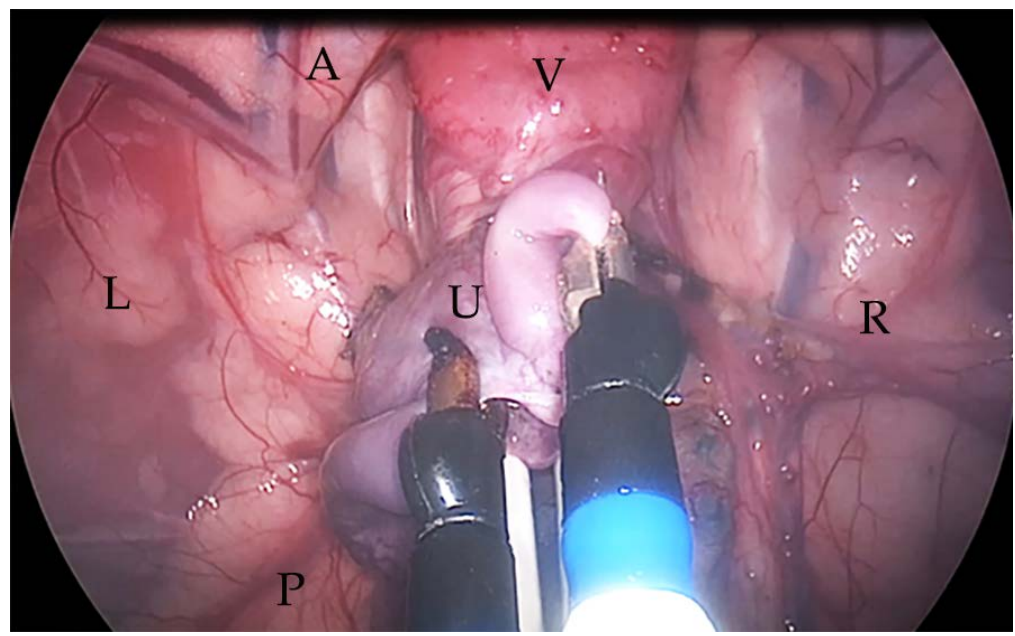

Figure 12. The uterus is amputated supracervically and the arms are straightened to remove the uterus. 


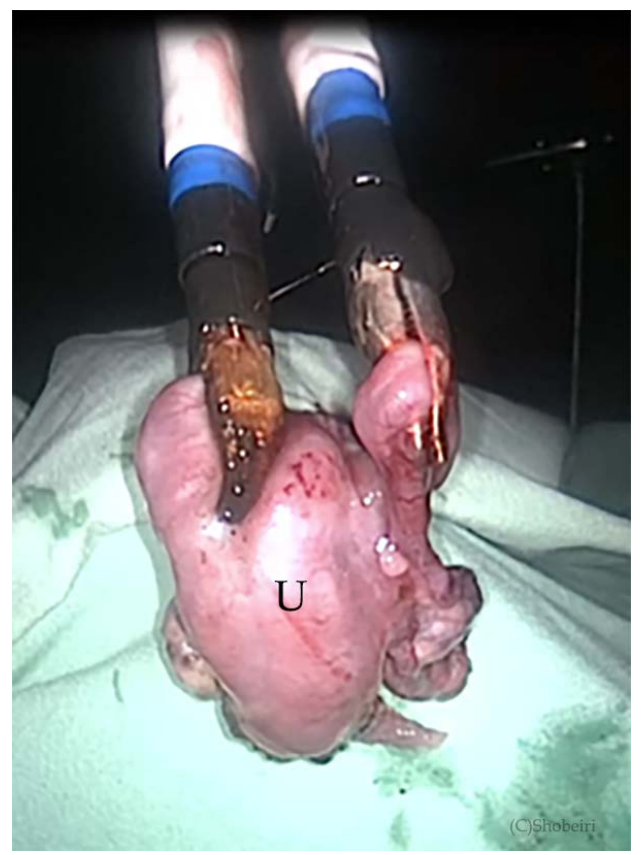

Figure 13. The uterus is extracted through the trocar. Legends: A: Anterior, Cu: Cul-de-sac, In: Insert, IP: Infundibulopelvic ligaments, L: Left, LO: Left Ovary, P: Posterior, R: Right, RO: Right Ovary, Sa: Sacrum: Si: Sigmoid colon; Tr: Trocar, Tu: Tube, U: Uterus, USL: Uterosacral ligaments, V: Vagina.

This novel robotic system allows the surgeon to approach the pelvic cavity in a scarless mater, as in the vNOTES laparoscopic technique. There are two main advantages for this robotic system over laparoscopic vNOTES surgeries. The first and most significant advantage is the $360^{\circ}$ articulation humanoid-shaped arms that allow access to areas unreachable when using the straight tools in laparoscopic vNOTES surgeries. The second notable advantage of this robot is the ability to work in the pelvis in a cranial to caudal orientation, just like the conservative abdominal laparoscopic surgeries, rather than the opposite orientation in vNOTES surgeries. The robotic system is built to allow the surgeon to view and explore the entire peritoneal cavity, including the uterine walls and the peritoneum covering the surface of the bladder and broad ligaments. In this study, we aimed to demonstrate the direct entry of the device into the Pouch of Douglas. In order to visualize the entry, we needed to insert an abdominal $5 \mathrm{~mm}$ laparoscopic port for the laparoscopic camera. Another $5 \mathrm{~mm}$ port was inserted abdominally for a laparoscopic grasper that helped with the manipulation of the uterus. Surely, the abdominal incisions diminish the advantages of the vNOTES approach, however, they were needed for the purpose of this study and simulated the presence of the in-built robotic camera arm and the in-built uterine manipulator that are present in the later versions of the Hominis ${ }^{\mathrm{Tm}}$ robotic system. The designated vaginal trocar for a direct entry into the Pouch of Douglas appears to be well designed to prevent bowel injury, however, further studies are needed to prove its safety. 


\section{Conclusion}

In conclusion, a supracervical hysterectomy via a vaginal approach using the novel vaginal Hominis ${ }^{\text {Tu }}$ robotic system was found to be feasible in an Ovine model. The robotic system has easy docking, short setup time and superior surgical and ergonomics capabilities. Further development and refinement of this system are underway in order to enhance the advantages of a single port scarless surgery.

\section{Acknowledgements}

The authors would like to recognize INOVA Advanced Surgical Technology \& Education Center and Mr. Larry Walker for allowing the evaluation of the robotic trocar in the dry lab.

\section{Conflicts of Interest}

The authors declare no conflicts of interest regarding the publication of this paper.

\section{References}

[1] Gettman, M.T., Box, G., Averch, T., et al. (2008) Consensus Statement on Natural Orifice Transluminal Endoscopic Surgery and Single-Incision Laparoscopic Surgery: Heralding a New Era in Urology? European Urology, 53, 1117-1120. https://doi.org/10.1016/j.eururo.2008.02.006

[2] Sawyer, M., Box, G., Averch, T., et al. (2008) Nomenclature of Natural Orifice Translumenal Endoscopic Surgery $\left(\right.$ NOTES $^{\mathrm{TM}}$ ) and Laparoendoscopic Single-Site Surgery (LESS) Procedures in Urology. Journal of Endourology, 22, 2575-2581. https://doi.org/10.1089/end.2008.0471

[3] McGee, M.F., Rosen, M.J., Marks, J., et al. (2006) A Primer on Natural Orifice Transluminal Endoscopic Surgery: Building a New Paradigm. Literature Review in Surgical Innovation, 13, 86-93. https://doi.org/10.1177/1553350606290529

[4] Yoshiki, N. (2017) Review of Transvaginal Natural Orifice Transluminal Endoscopic Surgery in Gynecology. Gynecology and Minimally Invasive Therapy, 6, 1-5. https://doi.org/10.1016/j.gmit.2016.11.007

[5] Lee, C.L., Wu, K.Y., Su, H., Ueng, S.H. and Yen, C.F. (2012) Transvaginal Natural-Orifice Transluminal Endoscopic Surgery (NOTES) in Adnexal Procedures. Journal of Minimally Invasive Gynecology, 19, 509-513. https://doi.org/10.1016/j.jmig.2012.02.005

[6] Ahn, K.H., Song, J.Y., Kim, S.H., Lee, K.W. and Kim, T. (2012) Transvaginal Single-Port Natural Orifice Transluminal Endoscopic Surgery for Benign Uterine Adnexal Pathologies. Journal of Minimally Invasive Gynecology, 19, 631-635. https://doi.org/10.1016/j.jmig.2012.04.001

[7] Yang, Y.S., Hur, M.H., Oh, K.Y. and Kim, S.Y. (2013) Transvaginal Natural Orifice Transluminal Endoscopic Surgery for Adnexal Masses. Journal of Obstetrics and Gynaecology Research, 39, 1604-1609. https://doi.org/10.1111/jog.12108

[8] Baekelandt, J. (2018) Transvaginal Natural Orifice Transluminal Endoscopic Surgery: A New Approach to Ovarian Cystectomy. Fertility and Sterility, 109, 366. https://doi.org/10.1016/j.fertnstert.2017.10.037 
[9] Su, H., Yen, C.F., Wu, K.Y., Han, C.M. and Lee, C.L. (2012) Hysterectomy via Transvaginal Natural Orifice Transluminal Endoscopic Surgery (NOTES): Feasibility of an Innovative Approach. Taiwanese Journal of Obstetrics \& Gynecology, 51, 217-221. https://doi.org/10.1016/j.tjog.2012.04.009

[10] Baekelandt, J. (2015) Total Vaginal NOTES Hysterectomy: A New Approach to Hysterectomy. Journal of Minimally Invasive Gynecology, 22, 1088-1094. https://doi.org/10.1016/j.jmig.2015.05.015

[11] Targarona, E.M. (2010) Natural Orifice Transluminal Endoscopic Surgery: The Transvaginal Route Moving Forward from Cholecystectomy. World Journal of Gastrointestinal Surgery, 2, 179. https://doi.org/10.4240/wjgs.v2.i6.179

[12] Hackethal, A., Ionesi-Pasacica, J., Eskef, K., Oehmke, F., Münstedt, K. and Tinneberg, H.R. (2011) Transvaginal NOTES with Semi-Rigid and Rigid Endoscopes That Allow Adjustable Viewing Angles. Archives of Gynecology and Obstetrics, 283, 131-132. https://doi.org/10.1007/s00404-010-1430-4

[13] Lee, C.L., Wu, K.Y., Su, H., Han, C.M., Huang, C.Y. and Yen, C.F. (2015) Robot-Assisted Natural Orifice Transluminal Endoscopic Surgery for Hysterectomy. Taiwanese Journal of Obstetrics \& Gynecology, 54, 761-765. https://doi.org/10.1016/j.tjog.2015.08.023

[14] Kerbage, Y., Cosson, M., Hubert, T. and Giraudet, G. (2017) Multiparous Ewe as a Model for Teaching Vaginal Hysterectomy Techniques. Obstetrics \& Gynecology, 130, 1276-1278. https://doi.org/10.1097/AOG.0000000000002363

[15] Kerbage, Y., Giraudet, G., Rubod, C., Garabedian, C., Rivaux, G. and Cosson, M. (2017) Feasibility and Benefits of the Ewe as a Model for Vaginal Surgery Training. International Urogynecology Journal, 28, 1573-1577. https://doi.org/10.1007/s00192-017-3313-8

[16] Urbankova, I., Vdoviakova, K., Rynkevic, R., et al. (2017) Comparative Anatomy of the Ovine and Female Pelvis. Gynecologic and Obstetric Investigation, 82, 582-591. https://doi.org/10.1159/000454771

[17] Mansoor, A., Curinier, S., Campagne-Loiseau, S., Platteeuw, L., Jacquetin, B. and Rabischong, B. (2017) Development of an Ovine Model for Training in Vaginal Surgery for Pelvic Organ Prolapse. International Urogynecology Journal, 28, 1595-1597. https://doi.org/10.1007/s00192-017-3292-9

[18] Urbankova, I., Callewaert, G., Sindhwani, N., et al. (2017) Transvaginal Mesh Insertion in the Ovine Model. Journal of Visualized Experiments, No. 125, e55706. https://doi.org/10.3791/55706

[19] Ramirez, E.R., Ramirez, D.K., Pillari, V.T., Vasquez, H. and Ramirez, H.A. (2008) Modified Uterine Transplant Procedure in the Sheep Model. Journal of Minimally Invasive Gynecology, 15, 311-314. https://doi.org/10.1016/j.jmig.2008.01.014

[20] D’Albuquerque, L., Ejzenberg, D., Santos, R., et al. (2017) Sheep Model for Uterine Transplantation: The Best Option before Starting a Human Program. Clinics, 72, 178-182. https://doi.org/10.6061/clinics/2017(03)08

[21] Wei, L., Xue, T., Yang, H., et al. (2013) Modified Uterine Allotransplantation and Immunosuppression Procedure in the Sheep Model. PLoS ONE, 8, e81300. https://doi.org/10.1371/journal.pone.0081300

[22] Saso, S., Petts, G., Thum, M.Y., et al. (2015) Achieving Uterine Auto-Transplantation in a Sheep Model Using Iliac Vessel Anastomosis: A Short-Term Viability Study. Acta Obstetricia et Gynecologica Scandinavica, 94, 245-252. https://doi.org/10.1111/aogs.12550 
[23] Saso, S., Petts, G., Thum, M.Y., Corless, D., Boyd, M., Noakes, D., Del Priore, G., Ghaem-Maghami, S. and Smith, J.R. (2015) Achieving Uterine Auto-Transplantation in a Sheep Model Using Iliac Vessel Anastomosis: a Short-Term Viability Study. Acta Obstetricia et Gynecologica Scandinavica, 94, 245-252.

[24] Aaronson, O.S., Tulipan, N.B., Cywes, R., et al. (2002) Robot-Assisted Endoscopic Intrauterine Myelomeningocele Repair: A Feasibility Study. Pediatric Neurosurgery, 36, 85-89. https://doi.org/10.1159/000048358

[25] After, O., Versus, T. and Abdominal, S. (2002) Outcomes after Total versus Subtotal Abdominal Hysterectomy. The New England Journal of Medicine, 347, 1318-1325. http://www.nejm.org/doi/full/10.1056/NEJMoa013336\#t=article https://doi.org/10.1056/NEJMoa013336

[26] Nesbitt-Hawes, E.M., Maley, P.E., Won, H.R., et al. (2013) Laparoscopic Subtotal Hysterectomy: Evidence and Techniques. Journal of Minimally Invasive Gynecology, 20, 424-434. https://doi.org/10.1016/j.jmig.2013.01.009

[27] Gimbel, H., Zobbe, V., Andersen, B.M., Filtenborg, T., Gluud, C. and Tabor, A. (2003) Randomised Controlled Trial of Total Compared with Subtotal Hysterectomy with One-Year Follow Up Results. BJOG: An International Journal of Obstetrics \&amp; Gynaecology, 110, 1088-1098. https://doi.org/10.1111/j.1471-0528.2003.02395.x

[28] Learman, L.A., Summitt, R.L., Varner, R.E., et al. (2003) A Randomized Comparison of Total or Supracervical Hysterectomy: Surgical Complications and Clinical Outcomes. Obstetrics \& Gynecology, 102, 453-462. https://doi.org/10.1016/S0029-7844(03)00664-1

[29] Gorlero, F., Lijoi, D., Biamonti, M., et al. (2008) Hysterectomy and Women Satisfaction: Total versus Subtotal Technique. Archives of Gynecology and Obstetrics, 278, 405-410. https://doi.org/10.1007/s00404-008-0615-6

[30] Donnez, O., Jadoul, P., Squifflet, J. and Donnez, J. (2009) A Series of 3190 Laparoscopic Hysterectomies for Benign Disease from 1990 to 2006: Evaluation of Complications Compared with Vaginal and Abdominal Procedures. BJOG: An International Journal of Obstetrics \&amp; Gynaecology, 492-500. https://doi.org/10.1111/j.1471-0528.2008.01966.x

[31] Bojahr, B., Raatz, D., Schonleber, G., Abri, C. and Ohlinger, R. (2006) Perioperative Complication Rate in 1706 Patients after a Standardized Laparoscopic Supracervical Hysterectomy Technique. Journal of Minimally Invasive Gynecology, 13, 183-189. https://doi.org/10.1016/j.jmig.2006.01.010

[32] Gimbel, H. (2007) Total or Subtotal Hysterectomy for Benign Uterine Diseases? A Meta-Analysis. Acta Obstetricia et Gynecologica Scandinavica, 86, 133-144. https://doi.org/10.1080/00016340601024716

[33] Lyons, T. (2007) Laparoscopic Supracervical versus Total Hysterectomy. Journal of Minimally Invasive Gynecology, 14, 275-277. https://doi.org/10.1016/j.jmig.2006.10.032

[34] El-Mowafi, D., Madkour, W., Lall, C. and Wenger, J.M. (2004) Laparoscopic Supracervical Hysterectomy versus Laparoscopic-Assisted Vaginal Hysterectomy. Journal of Minimally Invasive Gynecology, 11, 175-180. https://doi.org/10.1016/S1074-3804(05)60194-6

[35] Flory, N., Bissonnette, F., Amsel, R.T. and Binik, Y.M. (2006) The Psychosocial Outcomes of Total and Subtotal Hysterectomy: A Randomized Controlled Trial. The Journal of Sexual Medicine, 3, 483-491. https://doi.org/10.1111/j.1743-6109.2006.00229.x 
[36] Robert, M., Soraisham, A. and Sauve, R. (2008) Postoperative Urinary Incontinence after Total Abdominal Hysterectomy or Supracervical Hysterectomy: A Metaanalysis. American Journal of Obstetrics \& Gynecology, 198, 264.e1-264.e5. https://doi.org/10.1016/j.ajog.2007.09.033

[37] Lethaby, A., Ivanova, V. and Johnson, N. (2010) Total versus Subtotal Hysterectomy for Benign Gynaecological Conditions. Obstetrics \& Gynecology, 110, 705-706. https://doi.org/10.1097/01.AOG.0000280283.83502.e8

[38] Sarmini, O.R., Lefholz, K. and Froeschke, H.P. (2005) A Comparison of Laparoscopic Supracervical Hysterectomy and Total Abdominal Hysterectomy Outcomes. Journal of Minimally Invasive Gynecology, 12, 121-124. https://doi.org/10.1016/j.jmig.2005.01.019

[39] Storm, H.H., Clemmensen, I.H., Manders, T. and Brinton, L.A. (1992) Supravaginal Uterine Amputation in Denmark 1978-1988 and Risk of Cancer. Gynecologic Oncology, 45, 198-201. https://doi.org/10.1016/0090-8258(92)90285-Q

[40] Ghomi, A., Hantes, J. and Lotze, E.C. (2005) Incidence of Cyclical Bleeding after Laparoscopic Supracervical Hysterectomy. Journal of Minimally Invasive Gynecology, 12, 201-205. https://doi.org/10.1016/j.jmig.2005.03.008

[41] Hilger, W.S. and Magrina, J.F. (2006) Removal of Pelvic Leiomyomata and Endometriosis Five Years after Supracervical Hysterectomy. Obstetrics \& Gynecology, 108, 772-774. https://doi.org/10.1097/01.AOG.0000209187.90019.d3

[42] Van Der Meulen, J.F., Pijnenborg, J.M.A., Boomsma, C.M., Verberg, M.F.G., Geomini, P.M.A.J. and Bongers, M.Y. (2016) Parasitic Myoma after Laparoscopic Morcellation: A Systematic Review of the Literature. BJOG: An International Journal of Obstetrics \& Gynaecology, 123, 69-75. https://doi.org/10.1111/1471-0528.13541

[43] Ordulu, Z., Dal Cin, P., Chong, W.W.S., et al. (2009) Disseminated Peritoneal Leiomyomatosis after Laparoscopic Supracervical Hysterectomy with Characteristic Molecular Cytogenetic Findings of Uterine Leiomyoma. Genes, Chromosomes and Cancer, 49, 1152-1160. https://doi.org/10.1002/gcc.20824

[44] Worthington-Kirsch, R.L. and Hutchins, F.L. (2001) Retained Myoma Fragment after LASH Procedure. Clinical Radiology, 56, 777-778. https://doi.org/10.1053/crad.1999.0288

[45] Lee, C.L., Huang, C.Y., Wu, K.Y., Hu, Y.F., Yen, C.F. and Han, C.M. (2014) Natural Orifice Transvaginal Endoscopic Surgery Myomectomy: An Innovative Approach to Myomectomy. Gynecology and Minimally Invasive Therapy, 3, 127-130. https://doi.org/10.1016/j.gmit.2014.09.001

[46] Lee, C.L., Wu, K.Y., Tsao, F.Y., et al. (2014) Natural Orifice Transvaginal Endoscopic Surgery for Endometrial Cancer. Gynecology and Minimally Invasive Therapy, 3, 89-92. https://doi.org/10.1016/j.gmit.2014.08.004

[47] Han, C.M., Wu, K.Y., Su, H., et al. (2014) Feasibility of Transumbilical Single-Port Laparoscopic Hysterectomy Using Conventional Instruments. Gynecology and Minimally Invasive Therapy, 3, 47-49. https://doi.org/10.1016/j.gmit.2014.05.002

[48] Han, C.-M., Lee, C.-L., Huang, L., et al. (2018) Natural Orifice Transluminal Endoscopic Surgery (NOTES) Subtotal Hysterectomy: A Feasibility Study. Taiwanese Journal of Obstetrics \& Gynecology, 57, 355-359.

https://doi.org/10.1016/j.tjog.2018.04.005 